\title{
Generalizations of the Jensen functional involving diamond integrals via Abel-Gontscharoff interpolation
}

\author{
Rabia Bibi ${ }^{1 *}$, Ammara Nosheen², Shanaz Bano ${ }^{2}$ and Josip Pečarić ${ }^{3}$
}

"Correspondence:

emaorr@gmail.com

${ }^{1}$ Department of Mathematics,

Abbottabad University of Science

and Technology, Havelian,

Abbottabad, Pakistan

Full list of author information is

available at the end of the article

\begin{abstract}
In this paper we obtain several refinements of the Jensen inequality on time scales by generalizing Jensen's functional for $n$-convex functions. We also investigate the bounds for the identities related to the new improvements obtained.
\end{abstract}

Keywords: Time-scales calculus; Abel-Gontscharoff's interpolation polynomial; Jensen's inequality; Green function

\section{Introduction}

Time-scales theory is a well-established theory, where a time scale is a nonempty closed subset of the real numbers. This theory is a unification of discrete and continuous analysis. It was first initiated by Stefen Hilger in 1988 and then several books and research papers appeared, e.g., see $[6,7]$ for the basic calculus on time scales. Delta and nabla integrals are the basic integrals on time scales. Then, diamond- $\alpha(\alpha \in(0,1))$ integrals were introduced [18] as a convex combination of delta and nabla integrals. In 2015, diamond integrals were introduced as a generalization of all time-scales integrals including delta, nabla and diamond- $\alpha$ integrals, see [8]. Therefore throughout this paper we write our results for diamond integrals, but these results are also satisfied for delta, nabla and diamond- $\alpha$ integrals. Also, note that our results hold for sums and integrals since sums and integrals are specific examples of time-scales integrals.

As we obtain our results for diamond integrals, we assume throughout in this paper that the basic notions of the time scales are understood. Consider the forward jump operator $\sigma: \mathbb{T} \rightarrow \mathbb{R}$ and the backward jump operator $\rho: \mathbb{T} \rightarrow \mathbb{R}$. Then, the gamma function, $\gamma:$ $\mathbb{T} \rightarrow \mathbb{R}$, is defined by

$$
\gamma(b)=\lim _{s \rightarrow b} \frac{\sigma(b)-s}{\sigma(b)+2 b-2 s-\rho(b)} .
$$

(c) The Author(s) 2022. This article is licensed under a Creative Commons Attribution 4.0 International License, which permits use, sharing, adaptation, distribution and reproduction in any medium or format, as long as you give appropriate credit to the original author(s) and the source, provide a link to the Creative Commons licence, and indicate if changes were made. The images or other third party material in this article are included in the article's Creative Commons licence, unless indicated otherwise in a credit line to the material. If material is not included in the article's Creative Commons licence and your intended use is not permitted by statutory regulation or exceeds the permitted use, you will need to obtain permission directly from the copyright holder. To view a copy of this licence, visit http://creativecommons.org/licenses/by/4.0/. 
This function is used to define the diamond integrals. Clearly,

$$
\gamma(b)= \begin{cases}\frac{1}{2}, & \text { if } b \text { is dense } \\ \frac{\sigma(b)-b}{\sigma(b)-\rho(b)}, & \text { if } b \text { is not dense }\end{cases}
$$

and $0 \leq \gamma(b) \leq 1$.

\subsection{Diamond integral}

Let $e, f \in \mathbb{T}(e<f)$. For the function, $\mathfrak{J}: \mathbb{T} \rightarrow \mathbb{R}$, the diamond integral (or $\diamond$-integral) of $\mathfrak{J}$ from $e$ to $f\left(\right.$ or on $[e, f]_{\mathbb{T}}$ ) is given by

$$
\int_{e}^{f} \mathfrak{J}(b) \diamond b=\int_{e}^{f} \gamma(b) \mathfrak{J}(b) \Delta b+\int_{e}^{f}(1-\gamma(b)) \mathfrak{J}(b) \nabla b
$$

provided $\gamma \mathfrak{J}$ is $\Delta$-integrable and $(1-\gamma) \mathfrak{J}$ is $\nabla$-integrable on $[e, f]_{\mathbb{T}}$. If $\mathfrak{J}$ is $\nabla$-integrable $\forall$ $e, f \in \mathbb{T}$, then we say that it is diamond integrable (or $\diamond$-integrable) on $[e, f]_{\mathbb{T}}$. Note that if $\mathbb{T}=\mathbb{R}$, then

$$
\int_{e}^{f} \mathfrak{J}(b) \diamond b=\int_{e}^{f} \mathfrak{J}(b) d b ;
$$

if $\mathbb{T}=\mathbb{Z}$, then

$$
\int_{e}^{f} \mathfrak{J}(b) \diamond b=\frac{1}{2}\left(\sum_{b=e}^{f-1} \mathfrak{J}(b)+\sum_{b=e+1}^{f} \mathfrak{J}(b)\right) ;
$$

if $\mathbb{T}=h \mathbb{Z}$, where $h>0$, then

$$
\int_{e}^{f} \mathfrak{J}(b) \diamond b=\frac{h}{2}\left(\alpha \sum_{b=e / h}^{f / h-1} \mathfrak{J}(b h)+(1-\alpha) \sum_{b=e / h+1}^{f / h} \mathfrak{J}(b h)\right) ;
$$

if $\mathbb{T}=q^{\mathbb{N}_{0}}$, where $q>1$, then

$$
\int_{e}^{f} \mathfrak{J}(b) \diamond b=\frac{q-1}{q+1}\left(\sum_{b=\log _{q}(e)}^{\log _{q}(f)-1} q^{b} \mathfrak{J}\left(q^{b}\right)+\sum_{b=\log _{q}(e)+1}^{\log _{q}(f)} q^{b} \mathfrak{J}\left(q^{b}\right)\right) .
$$

Throughout mathematics and specifically in mathematical analysis Jensen's inequality for convex functions has great importance [3,12-15]. The number of research papers where Jensen's inequality is used is not countable. Several generalizations and refinements of Jensen's inequality for time-scales integrals can also be found in the literature (see $[1,5$, $6,11,16,18])$. For diamond integrals, Jensen's inequality is generalized in 2017, see [4].

Theorem 1.1 (Jensen's inequality) Let $e, f \in \mathbb{T}$ with $e \leq f, \mathfrak{J} \in C\left([e, f]_{\mathbb{T}}, \mathbb{R}\right)$ and $g \in$ $C\left([e, f]_{\mathbb{T}}, E\right)$. Also, assume $\phi \in C(E, \mathbb{R})$ is a convex function satisfying $\int_{e}^{f} \mathfrak{J}(b) \diamond b \neq 0$, where $E=\left[\mathfrak{o}_{1}, \mathfrak{o}_{2}\right]$ such that $\mathfrak{o}_{1}=\min _{b \in[e, f]_{\mathbb{T}}} g(b), \mathfrak{o}_{2}=\max _{b \in[e, f]_{\mathbb{T}}} g(b)$. Then, we have

$$
\phi\left(\frac{\int_{e}^{f}|\mathfrak{J}(b)| g(b) \diamond b}{\int_{e}^{f}|\mathfrak{J}(b)| \diamond b}\right) \leq \frac{\int_{e}^{f}|\mathfrak{J}(b)| \phi(g(b)) \diamond b}{\int_{e}^{f}|\mathfrak{J}(b)| \diamond b} .
$$


From Jensen's inequality, we obtain Jensen's functional:

$$
J(\phi)=\frac{\int_{e}^{f}|\mathfrak{J}(b)| \phi(g(b)) \diamond b}{\int_{e}^{f}|\mathfrak{J}(b)| \diamond b}-\phi\left(\frac{\int_{e}^{f}|\mathfrak{J}(b)| g(b) \diamond b}{\int_{e}^{f}|\mathfrak{J}(b)| \diamond b}\right) .
$$

Remark 1.2 For $\mathbb{T}=\mathbb{R}$, Jensen's functional becomes

$$
J(\phi)=\frac{\int_{e}^{f}|\mathfrak{J}(b)| \phi(g(b)) d b}{\int_{e}^{f}|\mathfrak{J}(b)| d b}-\phi\left(\frac{\int_{e}^{f}|\mathfrak{J}(b)| g(b) d b}{\int_{e}^{f}|\mathfrak{J}(b)| d b}\right) .
$$

If $\mathbb{T}=\mathbb{Z}$, then

$$
\begin{aligned}
J(\phi)= & \frac{\sum_{b=e}^{f-1}|\mathfrak{J}(b)| \phi(g(b))+\sum_{b=e+1}^{f}|\mathfrak{J}(b)| \phi(g(b))}{\sum_{b=e}^{f-1}|\mathfrak{J}(b)|+\sum_{b=e+1}^{f}|\mathfrak{J}(b)|} \\
& -\phi\left(\frac{\sum_{b=e}^{f-1}|\mathfrak{J}(b)| g(b)+\sum_{b=e+1}^{f}|\mathfrak{J}(b)| g(b)}{\sum_{b=e}^{f-1}|\mathfrak{J}(b)|+\sum_{b=e}^{f-1}|\mathfrak{J}(b)|}\right) .
\end{aligned}
$$

If $\mathbb{T}=h \mathbb{Z}$, where $h>0$, then

$$
\begin{aligned}
J(\phi)= & \frac{\alpha \sum_{b=e / h}^{f / h-1}|\mathfrak{J}(b h)| \phi(g(b h))+(1-\alpha) \sum_{b=e / h+1}^{f / h}|\mathfrak{J}(b h)| \phi(g(b h))}{\alpha \sum_{b=e / h}^{f / h-1}|\mathfrak{J}(b h)|+(1-\alpha) \sum_{b=e / h+1}^{f / h}|\mathfrak{J}(b h)|} \\
& -\phi\left(\frac{\alpha \sum_{b=e / h}^{f / h-1}|\mathfrak{J}(b h)| g(b h)+(1-\alpha) \sum_{b=e / h+1}^{f / h}|\mathfrak{J}(b h)| g(b h)}{\alpha \sum_{b=e / h}^{f / h-1}|\mathfrak{J}(b h)|+(1-\alpha) \sum_{b=e / h+1}^{f / h}|\mathfrak{J}(b h)|}\right) .
\end{aligned}
$$

If $\mathbb{T}=q^{\mathbb{N}_{0}}$, where $q>1$, then

$$
\begin{aligned}
J(\phi)= & \frac{\sum_{b=\log _{q}(e)}^{\log _{q}(f)-1} q^{b}\left|\mathfrak{J}\left(q^{b}\right)\right| \phi\left(g\left(q^{b}\right)\right)+\sum_{b=\log _{q}(e)+1}^{\log _{q}(f)} q^{b}\left|\mathfrak{J}\left(q^{b}\right)\right| \phi\left(g\left(q^{b}\right)\right)}{\sum_{b=\log _{q}(e)}^{\log _{q}(f)-1} q^{b}\left|\mathfrak{J}\left(q^{b}\right)\right|+\sum_{b=\log _{q}(e)+1}^{\log _{q}(f)} q^{b}\left|\mathfrak{J}\left(q^{b}\right)\right|} \\
& -\phi\left(\frac{\sum_{b=\log _{q}(e)}^{\log _{q}(f)-1} q^{b}\left|\mathfrak{J}\left(q^{b}\right)\right| g\left(q^{b}\right)+\sum_{b=\log _{q}(e)+1}^{\log _{q}(f)} q^{b}\left|\mathfrak{J}\left(q^{b}\right)\right| g\left(q^{b}\right)}{\sum_{b=\log _{q}(e)}^{\log _{q}(f)-1} q^{b}\left|\mathfrak{J}\left(q^{b}\right)\right|+\sum_{b=\log _{q}(e)+1}^{\log _{q}(f)} q^{b}\left|\mathfrak{J}\left(q^{b}\right)\right|}\right) .
\end{aligned}
$$

Remark 1.3 From Jensen's inequality it is clear that $J(\phi) \geq 0$ for the family of convex functions and $J(\phi)=0$ for $\phi(b)=b$ or when $\phi$ is a constant function.

A function $\phi:[e, f] \rightarrow \mathbb{R}$ is called $n$-convex $(n \geq 0)$ (see [17]), if for all choices of $(n+1)$ different points $b_{0}, \ldots, b_{n} \in[e, f]$, we have the $n$ th-order divided difference nonnegative, i.e., $\left[b_{0}, \ldots, b_{n} ; \phi\right] \geq 0$.

\section{Generalization of Jensen's inequality}

Let $n, u \in \mathbb{N}, n \geq 2,0 \leq u \leq n-1, \phi \in C^{n}\left(\left[\mathfrak{o}_{1}, \mathfrak{o}_{2}\right]\right)$ and $G_{n}(r, q)$ be the Green function defined as,

$$
G_{n}(r, q)=\frac{1}{(n-1) !} \begin{cases}\sum_{\mathfrak{z}=0}^{l}\left(\begin{array}{ll}
n-1 \\
\mathfrak{z}
\end{array}\right)\left(r-\mathfrak{o}_{1}\right)^{\mathfrak{z}}\left(\mathfrak{o}_{1}-q\right)^{n-\mathfrak{z}-1}, & \mathfrak{o}_{1} \leq q \leq r, \\
-\sum_{\mathfrak{z}=u+1}^{n-u}\left(\begin{array}{c}
n-1 \\
\mathfrak{z}
\end{array}\right)\left(r-\mathfrak{o}_{1}\right)^{\mathfrak{z}}\left(\mathfrak{o}_{1}-q\right)^{n-\mathfrak{z}-1}, & r \leq q \leq \mathfrak{o}_{2}\end{cases}
$$


Then, for $\mathfrak{o}_{1} \leq r, q \leq \mathfrak{o}_{2}$, the following inequalities hold (see [2])

$$
\begin{aligned}
& (-1)^{n-u-1} \frac{\partial^{\mathfrak{z}} G_{n}(r, q)}{\partial r^{\mathfrak{z}}} \geq 0, \quad 0 \leq \mathfrak{z} \leq u, \\
& (-1)^{n-u} \frac{\partial^{\mathfrak{z}} G_{n}(r, q)}{\partial r^{\mathfrak{z}}} \geq 0, \quad u+1 \leq \mathfrak{z} \leq n-1 .
\end{aligned}
$$

Assume

$$
\begin{aligned}
T_{n-1}\left(\mathfrak{o}_{1}, \mathfrak{o}_{2}, r ; \phi\right)= & \sum_{\mathfrak{z}=0}^{u} \frac{\left(r-\mathfrak{o}_{1}\right)^{c}}{\mathfrak{z} !} \phi^{(\mathfrak{z})}\left(\mathfrak{o}_{1}\right) \\
& +\sum_{d=0}^{n-u-2}\left[\sum_{\mathfrak{z}=0}^{d} \frac{\left(r-\mathfrak{o}_{1}\right)^{u+1+\mathfrak{z}}\left(\mathfrak{o}_{1}-\mathfrak{o}_{2}\right)^{d-\mathfrak{z}}}{(u+1+\mathfrak{z}) !(d-\mathfrak{z}) !}\right] \phi^{(u+1+d)}\left(\mathfrak{o}_{2}\right),
\end{aligned}
$$

is the Abel-Gontscharoff interpolation polynomial of degree $n-1$, then we have

$$
\phi(r)=T_{n-1}\left(\mathfrak{o}_{1}, \mathfrak{o}_{2}, r ; \phi\right)+R(r ; \phi)
$$

[2] (see also [10, 19-22]), where the remainder is given by

$$
R(r ; \phi)=\int_{\mathfrak{o}_{1}}^{\mathfrak{o}_{2}} G_{n}(r, q) \phi^{n}(q) d q
$$

By using Abel-Gontscharoff's interpolating polynomial, we establish the following identity.

Theorem 2.1 Let $n, \mathfrak{u} \in \mathbb{N}, n \geq 2,0 \leq \mathfrak{u} \leq n-1$. If $g \in C\left([e, f]_{\mathbb{T}}, E\right), \phi \in C^{n}(E, \mathbb{R})$ is a convex function, and $\mathfrak{J} \in C\left([e, f]_{\mathbb{T}}, \mathbb{R}\right)$ such that $\int_{e}^{f} \mathfrak{J}(b) \diamond b>0$, then we have

$$
\begin{aligned}
J(\phi(g))= & \sum_{\mathfrak{z}=2}^{\mathfrak{u}} \frac{\phi^{(\mathfrak{z})}\left(\mathfrak{o}_{1}\right)}{\mathfrak{z} !} J\left(g(b)-\mathfrak{o}_{1}\right)^{\mathfrak{z}} \\
& +\sum_{d=0}^{n-\mathfrak{u}-2}\left[\sum_{\mathfrak{z}=0}^{d} \frac{(-1)^{d-\mathfrak{z}}\left(\mathfrak{o}_{2}-\mathfrak{o}_{1}\right)^{d-\mathfrak{z}} \phi^{(\mathfrak{u}+1+d)}\left(\mathfrak{o}_{2}\right)}{(\mathfrak{u}+1+\mathfrak{z}) !(d-\mathfrak{z}) !}\right] \\
& \times J\left(g(b)-\mathfrak{o}_{1}\right)^{(\mathfrak{u}+1+\mathfrak{z})} \\
& +\int_{\mathfrak{o}_{1}}^{\mathfrak{o}_{2}} J\left(G_{n}(g(b), q)\right) \phi^{n}(q) d q,
\end{aligned}
$$

where

$$
J\left(G_{n}(g(b), q)\right)=\frac{\int_{e}^{f}|\mathfrak{J}(b)| G_{n}(g(b), q) \diamond b}{\int_{e}^{f}|\mathfrak{J}(b)| \diamond b}-G_{n}\left(\frac{\int_{e}^{f}|\mathfrak{J}(b)| g(b) \diamond b}{\int_{e}^{f}|\mathfrak{J}(b)| \diamond b}, q\right) .
$$


Proof By replacing $r$ with $g$ in equation (7) we obtain

$$
\begin{aligned}
\phi(g(b))= & \sum_{\mathfrak{z}=2}^{\mathfrak{u}} \frac{\left(g(b)-\mathfrak{o}_{1}\right)^{\mathfrak{z}}}{\mathfrak{z} !} \phi^{\mathfrak{z}}\left(\mathfrak{o}_{1}\right) \\
& +\sum_{d=0}^{n-\mathfrak{u}-2}\left[\sum_{\mathfrak{z}=0}^{d} \frac{\left(g(b)-\mathfrak{o}_{1}\right)^{\mathfrak{u}+1+\mathfrak{z}}\left(\mathfrak{o}_{1}-\mathfrak{o}_{2}\right)^{d-\mathfrak{z}}}{(\mathfrak{u}+1+\mathfrak{z}) !(d-\mathfrak{z}) !}\right] \phi^{(\mathfrak{u}+1+d)}\left(\mathfrak{o}_{2}\right) \\
& +\int_{\mathfrak{o}_{1}}^{\mathfrak{o}_{2}}\left(G_{n}(g(b), q)\right) \phi^{n}(q) d q .
\end{aligned}
$$

Now, (8) is followed by substituting (9) into (1) and using the linearity of $J($.$) .$

Remark 2.2 For different time scales, special cases of the inequality (8) can be deduced. For example, when $\mathbb{T}=\mathbb{R}$ the inequality (8) holds with Jensen's functional (2). Similarly, for $\mathbb{T}=\mathbb{Z}$ the inequality (8) holds with Jensen's functional (3); for $\mathbb{T}=h \mathbb{Z}$, where $h>0$, the inequality (8) holds with Jensen's functional (4); for $\mathbb{T}=q^{\mathbb{N} 0}$, where $q>1$, the inequality (8) holds with Jensen's functional (5).

The next theorem gives the generalization of Jensen's inequality for $n$-convex functions.

Theorem 2.3 Assume that all the conditions of Theorem 2.1 are satisfied and

$$
J\left(G_{n}(g(b), q)\right) \geq 0, \quad \text { for all } q \in\left[\mathfrak{o}_{1}, \mathfrak{o}_{2}\right] \text { and } n \geq 2 \text {. }
$$

If $\phi$ is n-convex such that $\phi^{n-1}$ is absolutely continuous, then

$$
\begin{aligned}
J(\phi(g)) \geq & \sum_{\mathfrak{z}=2}^{\mathfrak{u}} \frac{\phi^{(\mathfrak{z})}\left(\mathfrak{o}_{1}\right)}{\mathfrak{z} !} J\left(g(b)-\mathfrak{o}_{1}\right)^{\mathfrak{z}} \\
& +\sum_{d=0}^{n-\mathfrak{u}-2}\left[\sum_{\mathfrak{z}=0}^{d} \frac{(-1)^{d-\mathfrak{z}}\left(\mathfrak{o}_{2}-\mathfrak{o}_{1}\right)^{d-\mathfrak{z}} \phi^{(\mathfrak{u}+1+d)}\left(\mathfrak{o}_{2}\right)}{(\mathfrak{u}+1+\mathfrak{z}) !(d-\mathfrak{z}) !}\right] J\left(g(b)-\mathfrak{o}_{1}\right)^{(\mathfrak{u}+1+\mathfrak{z})} .
\end{aligned}
$$

Proof Since $\phi^{n-1}$ is absolutely continuous on $\left[\mathfrak{o}_{1}, \mathfrak{o}_{2}\right], \phi^{n}$ exists almost everywhere. Also, $\phi$ is $n$-convex, therefore $\phi^{n}(b) \geq 0$ for all $b \in\left[\mathfrak{o}_{1}, \mathfrak{o}_{2}\right]$ (see [17, page 16]). Hence, the inequality (10) follows from (8).

Theorem 2.4 Suppose that all the suppositions of Theorem 2.1 are satisfied such that $\phi$ is n-convex.

(i) If $\mathfrak{u}$ is odd and $n$ is even, or $n$ is odd and $\mathfrak{u}$ is even, then the inequality (10) is satisfied.

(ii) If the inequality (10) holds and the function

$$
\begin{aligned}
V(g(b))= & \sum_{\mathfrak{z}=0}^{\mathfrak{u}} \frac{\left(g(b)-\mathfrak{o}_{1}\right)^{\mathfrak{z}}}{\mathfrak{z} !} \phi^{\mathfrak{z}}\left(\mathfrak{o}_{1}\right) \\
& +\sum_{d=0}^{n-\mathfrak{u}-2}\left[\sum_{\mathfrak{z}=0}^{d} \frac{\left(g(b)-\mathfrak{o}_{1}\right)^{\mathfrak{u}+1+\mathfrak{z}}\left(\mathfrak{o}_{1}-\mathfrak{o}_{2}\right)^{d-\mathfrak{z}}}{(\mathfrak{u}+1+\mathfrak{z}) !(d-\mathfrak{z}) !}\right] \phi^{(\mathfrak{u}+1+d)}\left(\mathfrak{o}_{2}\right)
\end{aligned}
$$

is convex, then the right-hand side of $(10)$ is nonnegative and we have $J(\phi(g)) \geq 0$. 
Proof

(i) By using (6) for $\mathfrak{o}_{1} \leq r, q \leq \mathfrak{o}_{2}$, we have

$$
(-1)^{n-\mathfrak{u}-1} \frac{\partial^{2} G_{n}(r, q)}{\partial r^{2}} \geq 0 .
$$

Clearly, $\frac{\partial^{2} G_{n}(r, q)}{\partial r^{2}} \geq 0$ if $\mathfrak{u}$ is odd and $n$ is even, or $n$ is odd and $\mathfrak{u}$ is even. In this case, $G_{n}$ is convex with respect to the first variable and hence the inequality (11) is followed by Theorem 2.1.

(ii) Since $J(\phi)$ is linear, we can restate the right-hand side of $(10)$ as $J(V(g(b)))$ and hence by Remark 1.3 we obtain the nonnegativity of the right-hand side of (10).

In order to obtain more generalizations of Jensen's inequality, we also use the following Green function $G:\left[\mathfrak{o}_{1}, \mathfrak{o}_{2}\right] \times\left[\mathfrak{o}_{1}, \mathfrak{o}_{2}\right] \rightarrow \mathbb{R}$ such that $\mathfrak{o}_{1}, \mathfrak{o}_{2} \in \mathbb{R}, \mathfrak{o}_{1} \neq \mathfrak{o}_{2}$.

$$
G(q, r)= \begin{cases}\frac{\left(q-\mathfrak{o}_{2}\right)\left(r-\mathfrak{o}_{1}\right)}{\mathfrak{o}_{2}-\mathfrak{o}_{1}}, & \mathfrak{o}_{1} \leq r \leq q ; \\ \frac{\left(r-\mathfrak{o}_{2}\right)\left(q-\mathfrak{o}_{1}\right)}{\mathfrak{o}_{2}-\mathfrak{o}_{1}}, & q \leq r \leq \mathfrak{o}_{2} .\end{cases}
$$

The function $G$ has continuity and convexity with respect to $r$ and $q$. It is well known that (see [17]) for any convex function $\phi \in C^{2}\left(\left[\mathfrak{o}_{1}, \mathfrak{o}_{2}\right]\right)$, we have

$$
\phi(b)=\frac{\mathfrak{o}_{2}-b}{\mathfrak{o}_{2}-\mathfrak{o}_{1}} \phi\left(\mathfrak{o}_{1}\right)+\frac{b-\mathfrak{o}_{1}}{\mathfrak{o}_{2}-\mathfrak{o}_{1}} \phi\left(\mathfrak{o}_{2}\right)+\int_{\mathfrak{o}_{1}}^{\mathfrak{o}_{2}} G(b, r) \phi^{\prime \prime}(r) d r .
$$

Theorem 2.5 Let $n, \mathfrak{u} \in \mathbb{N}, n \geq 4,0 \leq \mathfrak{u} \leq n-1$. If $g \in C\left([e, f]_{\mathbb{T}}, E\right), \phi \in C^{n}(E, \mathbb{R})$ is a convex function, and $\mathfrak{J} \in C\left([e, f]_{\mathbb{T}}, \mathbb{R}\right)$ such that $\int_{e}^{f} \mathfrak{J}(b) \diamond b>0$, then we have

$$
\begin{aligned}
J(\phi(g))= & \sum_{\mathfrak{z}=0}^{\mathfrak{u}} \frac{\phi^{(\mathfrak{z}+2)}\left(\mathfrak{o}_{1}\right)}{\mathfrak{z} !} \int_{\mathfrak{o}_{1}}^{\mathfrak{o}_{2}} J(G(g(b), r))\left(r-\mathfrak{o}_{1}\right)^{\mathfrak{z}} d r \\
& +\sum_{d=0}^{n-\mathfrak{u}-4}\left[\sum_{\mathfrak{z}=0}^{d} \frac{(-1)^{d-\mathfrak{z}}\left(\mathfrak{o}_{2}-\mathfrak{o}_{1}\right)^{d-\mathfrak{z}} \phi^{(\mathfrak{u}+3+d)}\left(\mathfrak{o}_{2}\right)}{(\mathfrak{u}+1+\mathfrak{z}) !(d-\mathfrak{z}) !}\right] \\
& \times \int_{\mathfrak{o}_{1}}^{\mathfrak{o}_{2}} J(G(g(b), r))\left(r-\mathfrak{o}_{1}\right)^{(\mathfrak{u}+1+\mathfrak{z})} d r \\
& +\int_{\mathfrak{o}_{1}}^{\mathfrak{o}_{2}} \int_{\mathfrak{o}_{1}}^{\mathfrak{o}_{2}} J(G(g(b), r)) G_{n-2}(r, q) \phi^{n}(q) d q d r .
\end{aligned}
$$

Proof By using (12) and the linearity of $J$ we obtain

$$
J(\phi(g))=\int_{\mathfrak{o}_{1}}^{\mathfrak{o}_{2}} J(G(g(b), r)) \phi^{\prime \prime}(r) d r .
$$


From (7), $\phi^{\prime \prime}(r)$ becomes

$$
\begin{aligned}
\phi^{\prime \prime}(r)= & \sum_{\mathfrak{z}=0}^{\mathfrak{u}} \frac{\left(r-\mathfrak{o}_{1}\right)^{\mathfrak{z}}}{\mathfrak{z} !} \phi^{(\mathfrak{z}+2)}\left(\mathfrak{o}_{1}\right) \\
& +\sum_{d=0}^{n-\mathfrak{u}-4}\left[\sum_{\mathfrak{z}=0}^{d} \frac{\left(r-\mathfrak{o}_{1}\right)^{\mathfrak{u}+1+\mathfrak{z}}\left(\mathfrak{o}_{1}-\mathfrak{o}_{2}\right)^{\mathfrak{d}-\mathfrak{i}}}{(\mathfrak{u}+1+\mathfrak{z}) !(d-\mathfrak{z}) !}\right] \phi^{(\mathfrak{u}+3+d)}\left(\mathfrak{o}_{2}\right) \\
& +\int_{\mathfrak{o}_{1}}^{\mathfrak{o}_{2}} G_{n-2}(r, q) \phi^{n}(q) d q .
\end{aligned}
$$

Using (16) in (15), we obtain (13).

Theorem 2.6 Let

$$
\int_{\mathfrak{o}_{1}}^{\mathfrak{o}_{2}} J(G(g(b), r))\left(G_{n-2}(r, q)\right) d r \geq 0, \quad \forall q \in\left[\mathfrak{o}_{1}, \mathfrak{o}_{2}\right],
$$

with the assumptions of Theorem 2.5. If $\phi$ is n-convex such that $\phi^{n-1}$ is absolutely continuous, then

$$
\begin{aligned}
J(\phi(g)) \geq & \sum_{\mathfrak{z}=0}^{\mathfrak{u}} \frac{\phi^{(\mathfrak{z}+2)}\left(\mathfrak{o}_{1}\right)}{\mathfrak{z} !} \int_{\mathfrak{o}_{1}}^{\mathfrak{o}_{2}} J(G(g(b), r))\left(r-\mathfrak{o}_{1}\right)^{\mathfrak{z}} d r \\
& +\sum_{d=0}^{n-\mathfrak{u}-4}\left[\sum_{\mathfrak{z}=0}^{d} \frac{(-1)^{d-\mathfrak{z}}\left(\mathfrak{o}_{2}-\mathfrak{o}_{1}\right)^{d-\mathfrak{z}} \phi^{(\mathfrak{u}+3+d)}\left(\mathfrak{o}_{2}\right)}{(\mathfrak{u}+1+\mathfrak{z}) !(d-\mathfrak{z}) !}\right] \\
& \times \int_{\mathfrak{o}_{1}}^{\mathfrak{o}_{2}} J(G(g(b), r))\left(r-\mathfrak{o}_{1}\right)^{\mathfrak{u}+1+\mathfrak{z}} d r .
\end{aligned}
$$

Proof We can prove this theorem like Theorem 2.3, except here we use Theorem 2.5 instead of Theorem 2.1.

Theorem 2.7 Suppose that all the suppositions of Theorem 2.1 are satisfied such that $\phi$ is n-convex.

(i) If $(n=o d d, l=$ even $)$ or $(l=o d d, n=$ even $)$, then

$$
\begin{aligned}
J(\phi) \geq & \sum_{\mathfrak{z}=0}^{\mathfrak{u}} \frac{\phi^{(\mathfrak{z}+2)}\left(\mathfrak{o}_{1}\right)}{\mathfrak{z} !} \int_{\mathfrak{o}_{1}}^{\mathfrak{o}_{2}} J(G(g(b), r))\left(r-\mathfrak{o}_{1}\right)^{\mathfrak{z}} d r \\
& +\sum_{d=0}^{n-\mathfrak{u}-4}\left[\sum_{\mathfrak{z}=0}^{d} \frac{(-1)^{d-\mathfrak{z}}\left(\mathfrak{o}_{2}-\mathfrak{o}_{1}\right)^{d-\mathfrak{z}} \phi^{(\mathfrak{u}+3+d)}\left(\mathfrak{o}_{2}\right)}{(\mathfrak{u}+1+\mathfrak{z}) !(d-\mathfrak{z}) !}\right] \\
& \times \int_{\mathfrak{o}_{1}}^{\mathfrak{o}_{2}} J(G(g(b), r))\left(r-\mathfrak{o}_{1}\right)^{\mathfrak{u}+1+\mathfrak{z}} d r .
\end{aligned}
$$

Moreover, suppose that $\phi^{(\mathfrak{z}+2)}\left(\mathfrak{o}_{1}\right) \geq 0$ if $\mathfrak{z}=0,1,2, \ldots, \mathfrak{u}$ and $\phi^{(\mathfrak{u}+3+d)}\left(\mathfrak{o}_{2}\right) \geq 0$ if $d-\mathfrak{z}$ is even, and $\phi^{(\mathfrak{u}+3+d)}\left(\mathfrak{o}_{2}\right) \leq 0$ if $d-\mathfrak{z}$ is odd, for $\mathfrak{z}=0, \ldots, d$ and $d=0, \ldots, n-\mathfrak{u}-4$.

Then, the right-hand side of the inequality (17) becomes nonnegative.

(ii) If both $n$ and $l$ are odd or even simultaneously, then the reverse of (17) is valid.

Further, if $\phi^{(\mathfrak{z}+2)}\left(\mathfrak{o}_{1}\right) \leq 0$ for $\mathfrak{z}=0,1,2, \ldots, \mathfrak{u}$ and $\phi^{(\mathfrak{u}+3+d)}\left(\mathfrak{o}_{2}\right) \leq 0$ if $d-\mathfrak{z}$ is even, and 
$\phi^{(\mathfrak{u}+3+d)}\left(\mathfrak{o}_{2}\right) \geq 0$, if $d-\mathfrak{z}$ is odd, for $\mathfrak{z}=0, \ldots, d$ and $d=0, \ldots, n-\mathfrak{u}-4$, then the right-hand side of the reverse inequality becomes nonpositive.

Proof We can prove this theorem like Theorem 2.4.

Remark 2.8 As mentioned in Remark 2.2, we can deduce special cases for the results of this section for different time scales.

\section{Bounds concerning the identities for generalization of a Jensen-type inequality}

For our results of this section, we use the following two theorems given by Cerone and Dragomir [9].

Theorem 3.1 Let $k:\left[\mathfrak{o}_{1}, \mathfrak{o}_{2}\right] \rightarrow \mathbb{R}$ be a Lebesgue integrable function and $l:\left[\mathfrak{o}_{1}, \mathfrak{o}_{2}\right] \rightarrow \mathbb{R}$ be an absolutely continuous function with $\left(.-\mathfrak{o}_{1}\right)\left(\mathfrak{o}_{2}-.\right)\left[l^{\prime}\right]^{2} \in L\left[\mathfrak{o}_{1}, \mathfrak{o}_{2}\right]$. Then, we have

$$
|\varphi(k, l)| \leq \frac{1}{\sqrt{2}}[\varphi(k, k)]^{\frac{1}{2}} \frac{1}{\sqrt{\mathfrak{o}_{2}-\mathfrak{o}_{1}}}\left[\int_{\mathfrak{o}_{1}}^{\mathfrak{o}_{2}}\left(b-\mathfrak{o}_{1}\right)\left(\mathfrak{o}_{2}-b\right)\left[l^{\prime}(b)\right]^{2} d b\right]^{\frac{1}{2}}
$$

where

$$
\varphi(k, l)=\frac{1}{\mathfrak{o}_{2}-\mathfrak{o}_{1}} \int_{\mathfrak{o}_{1}}^{\mathfrak{o}_{2}} k(q) l(b) d b-\frac{1}{\mathfrak{o}_{2}-\mathfrak{o}_{1}} \int_{\mathfrak{o}_{1}}^{\mathfrak{o}_{2}} k(b) d b \cdot \frac{1}{\mathfrak{o}_{2}-\mathfrak{o}_{1}} \int_{\mathfrak{o}_{1}}^{\mathfrak{o}_{2}} l(b) d b .
$$

In the inequality (18), the constant $\frac{1}{\sqrt{2}}$ is the most suitable option.

Theorem 3.2 Assume that $k:\left[\mathfrak{o}_{1}, \mathfrak{o}_{2}\right] \rightarrow \mathbb{R}$ is monotonic nondecreasing on $\left[\mathfrak{o}_{1}, \mathfrak{o}_{2}\right]$ and $l:\left[\mathfrak{o}_{1}, \mathfrak{o}_{2}\right] \rightarrow \mathbb{R}$ is absolutely continuous with $k^{\prime} \in L_{\infty}\left[\mathfrak{o}_{1}, \mathfrak{o}_{2}\right]$. Then, we have the inequality

$$
|\varphi(k, l)| \leq \frac{1}{2\left(\mathfrak{o}_{2}-\mathfrak{o}_{1}\right)}\left\|k^{\prime}\right\|_{\infty} \int_{\mathfrak{o}_{1}}^{\mathfrak{o}_{2}}\left(b-\mathfrak{o}_{1}\right)\left(\mathfrak{o}_{2}-b\right) d l(b)
$$

The constant $\frac{1}{2}$ in the above equation is the most suitable option.

The following notations are used throughout this section,

$$
\xi(q)=J\left(G_{n}(b, q)\right) \quad q \in\left[\mathfrak{o}_{1}, \mathfrak{o}_{2}\right]
$$

and

$$
\varphi(\xi, \xi)=\frac{1}{\mathfrak{o}_{2}-\mathfrak{o}_{1}} \int_{\mathfrak{o}_{1}}^{\mathfrak{o}_{2}} \xi^{2}(q) d q-\left(\frac{1}{\mathfrak{o}_{2}-\mathfrak{o}_{1}} \int_{\mathfrak{o}_{1}}^{\mathfrak{o}_{2}} \xi(q) d q\right)^{2}
$$

By using the Čhebyšev functional (19) we obtain the following identity. 
Theorem 3.3 If $\left(.-\mathfrak{o}_{1}\right)\left(\mathfrak{o}_{2}-.\right)\left[\phi^{n+1}\right]^{2} \in L\left[\mathfrak{o}_{1}, \mathfrak{o}_{2}\right]$ with the assumptions of Theorem 2.1 , then we have

$$
\begin{aligned}
J(\phi(g))= & \sum_{\mathfrak{z}=2}^{\mathfrak{u}} \frac{\phi^{(\mathfrak{z})}\left(\mathfrak{o}_{1}\right)}{\mathfrak{z} !} J\left(g(b)-\mathfrak{o}_{1}\right)^{\mathfrak{z}} \\
& +\sum_{d=0}^{n-\mathfrak{u}-2}\left[\sum_{\mathfrak{z}=0}^{d} \frac{(-1)^{d-\mathfrak{z}}\left(\mathfrak{o}_{2}-\mathfrak{o}_{1}\right)^{d-\mathfrak{z}} \phi^{(\mathfrak{u}+1+d)}\left(\mathfrak{o}_{2}\right)}{(\mathfrak{u}+1+\mathfrak{z}) !(d-\mathfrak{z}) !}\right] J\left(g(b)-\mathfrak{o}_{1}\right)^{(\mathfrak{u}+1+\mathfrak{z})} \\
& +\frac{\phi^{n-1}\left(\mathfrak{o}_{2}\right)-\phi^{n-1}\left(\mathfrak{o}_{1}\right)}{\mathfrak{o}_{2}-\mathfrak{o}_{1}} \int_{\mathfrak{o}_{1}}^{\mathfrak{o}_{2}} \xi(q) d q+\kappa_{n}\left(\mathfrak{o}_{1}, \mathfrak{o}_{2} ; \phi\right),
\end{aligned}
$$

where

$$
\left|\kappa_{n}\left(\mathfrak{o}_{1}, \mathfrak{o}_{2} ; \phi\right)\right| \leq \sqrt{\frac{\mathfrak{o}_{2}-\mathfrak{o}_{1}}{2}}[\varphi(\xi, \xi)]^{\frac{1}{2}}\left|\int_{\mathfrak{o}_{1}}^{\mathfrak{o}_{2}}\left(q-\mathfrak{o}_{1}\right)\left(\mathfrak{o}_{2}-q\right)\left[\phi^{(n+1)}(q)\right]^{2} d q\right|^{\frac{1}{2}}
$$

Proof By replacing $k$ with $\xi$ and $l$ with $\phi^{n}$ in Theorem 3.1, we obtain

$$
\begin{aligned}
& \left|\frac{1}{\mathfrak{o}_{2}-\mathfrak{o}_{1}} \int_{\mathfrak{o}_{1}}^{\mathfrak{o}_{2}} \xi(q) \phi^{n}(q) d q-\frac{1}{\mathfrak{o}_{2}-\mathfrak{o}_{1}} \int_{\mathfrak{o}_{1}}^{\mathfrak{o}_{2}} \xi(q) d q \cdot \frac{1}{\mathfrak{o}_{2}-\mathfrak{o}_{1}} \int_{\mathfrak{o}_{1}}^{\mathfrak{o}_{2}} \phi^{n}(q) d q\right| \\
& \leq \frac{1}{\sqrt{2}}[\varphi(\xi, \xi)]^{\frac{1}{2}} \frac{1}{\sqrt{\mathfrak{o}_{2}-\mathfrak{o}_{1}}}\left|\int_{\mathfrak{o}_{1}}^{\mathfrak{o}_{2}}\left(q-\mathfrak{o}_{1}\right)\left(\mathfrak{o}_{2}-q\right)\left[\phi^{(n+1)}(q)\right]^{2} d q\right|^{\frac{1}{2}} .
\end{aligned}
$$

Therefore,

$$
\int_{\mathfrak{o}_{1}}^{\mathfrak{o}_{2}} \xi(q) \phi^{n}(q) d q=\frac{\phi^{n-1}\left(\mathfrak{o}_{2}\right)-\phi^{n-1}\left(\mathfrak{o}_{1}\right)}{\mathfrak{o}_{2}-\mathfrak{o}_{1}} \int_{\mathfrak{o}_{1}}^{\mathfrak{o}_{2}} \xi(q) d q+\kappa_{n}\left(\mathfrak{o}_{1}, \mathfrak{o}_{2} ; \phi\right)
$$

where the estimation (21) is satisfied by the remainder $\kappa_{n}\left(\mathfrak{o}_{2}, \mathfrak{o}_{1} ; \phi\right)$. Now, from identity (8) we obtain (20).

Our next theorem gives a Grüss-type inequality for diamond integrals.

Theorem 3.4 Let $0 \leq u \leq n-1$ and $\phi \in C^{n}\left(\left[\mathfrak{o}_{1}, \mathfrak{o}_{2}\right]\right)$, where $\phi^{n+1} \geq 0$ on $\left[\mathfrak{o}_{1}, \mathfrak{o}_{2}\right]$. Then, (20) is satisfied with

$$
\left|\kappa_{n}\left(\mathfrak{o}_{1}, \mathfrak{o}_{2} ; \phi\right)\right| \leq\left(\mathfrak{o}_{2}-\mathfrak{o}_{1}\right)\left\|\xi^{\prime}\right\|_{\infty}\left\{\frac{\phi^{n-1}\left(\mathfrak{o}_{2}\right)+\phi^{n-1}\left(\mathfrak{o}_{1}\right)}{2}-\frac{\phi^{n-2}\left(\mathfrak{o}_{2}\right)-\phi^{n-2}\left(\mathfrak{o}_{1}\right)}{\mathfrak{o}_{2}-\mathfrak{o}_{1}}\right\}
$$

Proof By replacing $k$ with $\xi$ and $l$ with $\phi^{n}$ in Theorem 3.2, we obtain

$$
\begin{aligned}
& \frac{1}{\mathfrak{o}_{2}-\mathfrak{o}_{1}} \int_{\mathfrak{o}_{1}}^{\mathfrak{o}_{2}} \xi(q) \phi^{n}(q) d q-\frac{1}{\mathfrak{o}_{2}-\mathfrak{o}_{1}} \int_{\mathfrak{o}_{1}}^{\mathfrak{o}_{2}} \xi(q) d q \cdot \frac{1}{\mathfrak{o}_{2}-\mathfrak{o}_{1}} \int_{\mathfrak{o}_{1}}^{\mathfrak{o}_{2}} \phi^{n}(q) d q \\
& \leq\left.\frac{1}{2\left(\mathfrak{o}_{2}-\mathfrak{o}_{1}\right)}|| \xi^{\prime}\right|_{\infty} \int_{\mathfrak{o}_{1}}^{\mathfrak{o}_{2}}\left(q-\mathfrak{o}_{1}\right)\left(\mathfrak{o}_{2}-q\right) \phi^{n+1}(q) d q .
\end{aligned}
$$


Since

$$
\begin{aligned}
\int_{\mathfrak{o}_{1}}^{\mathfrak{o}_{2}} & \left(q-\mathfrak{o}_{1}\right)\left(\mathfrak{o}_{2}-q\right) \phi^{n+1}(q) d q \\
\quad= & \int_{\mathfrak{o}_{1}}^{\mathfrak{o}_{2}}\left[2 q-\left(\mathfrak{o}_{1}+\mathfrak{o}_{2}\right)\right] \phi^{n}(q) d q \\
\quad= & \left(\mathfrak{o}_{2}-\mathfrak{o}_{1}\right)\left[\phi^{n-1}\left(\mathfrak{o}_{2}\right)+\phi^{n-1}\left(\mathfrak{o}_{1}\right)\right]-2\left[\phi^{n-2}\left(\mathfrak{o}_{2}\right)-\phi^{n-2}\left(\mathfrak{o}_{1}\right)\right],
\end{aligned}
$$

we deduce (22) by using the identity (8) and the inequality (23).

In the following theorem, we obtain an Ostrowski-type inequality for diamond integrals to generalize Jensen's inequality.

Theorem 3.5 Let $\mathfrak{h}, z \in[1, \infty]$ such that $\frac{1}{\mathfrak{h}}+\frac{1}{z}=1$. If $\left|\phi^{n}\right|^{\mathfrak{h}}:\left[\mathfrak{o}_{1}, \mathfrak{o}_{2}\right] \rightarrow \mathbb{R}$ is integrable for some $n \in \mathbb{N}$ and $n \geq 2$ with the assumptions of Theorem 2.1 , then we have

$$
\begin{aligned}
J(\phi) & -\sum_{\mathfrak{z}=0}^{\mathfrak{u}} \frac{\phi^{\mathfrak{z}}\left(\mathfrak{o}_{1}\right)}{\mathfrak{z} !} J\left(q-\mathfrak{o}_{1}\right)^{\mathfrak{z}} \\
& -\sum_{d=0}^{n-\mathfrak{u}-2}\left[\sum_{\mathfrak{z}=0}^{d} \frac{(-1)^{d-\mathfrak{z}}\left(\mathfrak{o}_{2}-\mathfrak{o}_{1}\right)^{d-\mathfrak{z}} \phi^{(\mathfrak{u}+1+d)}\left(\mathfrak{o}_{2}\right)}{(\mathfrak{u}+1+\mathfrak{z}) !(d-\mathfrak{z}) !}\right] J\left(q-\mathfrak{o}_{1}\right)^{(\mathfrak{u}+1+\mathfrak{z})} \\
\leq & \| \phi^{(n)}||_{\mathfrak{h}}\left(\int_{\mathfrak{o}_{1}}^{\mathfrak{o}_{2}}\left|J\left(G_{n}(g(b), q)\right)\right|^{z} d q\right)^{\frac{1}{z}} .
\end{aligned}
$$

The constant on the right-hand side of (24) is sharp for $1<\mathfrak{h} \leq \infty$ and the best possible for $\mathfrak{h}=1$.

Proof Utilizing identity (8) and after application of Hölder's inequality, we obtain

$$
\begin{aligned}
& J(\phi(g))-\sum_{\mathfrak{z}=2}^{\mathfrak{u}} \frac{\phi^{\mathfrak{z}}\left(\mathfrak{o}_{1}\right)}{\mathfrak{z} !} J\left(g(b)-\mathfrak{o}_{1}\right)^{\mathfrak{z}} \\
& \quad+\sum_{d=0}^{n-\mathfrak{u}-2}\left[\sum_{\mathfrak{z}=0}^{d} \frac{(-1)^{d-\mathfrak{z}}\left(\mathfrak{o}_{2}-\mathfrak{o}_{1}\right)^{d-\mathfrak{z}} \phi^{(\mathfrak{u}+1+d)}\left(\mathfrak{o}_{2}\right)}{(\mathfrak{u}+1+\mathfrak{z}) !(d-\mathfrak{z}) !}\right] J\left(g(b)-\mathfrak{o}_{1}\right)^{(\mathfrak{u}+1+\mathfrak{z})} \\
& =\left|\int_{\mathfrak{o}_{1}}^{\mathfrak{o}_{2}} \xi(q) \phi^{n}(q) d q\right| \leq\left\|\phi^{(n)}\right\|_{\mathfrak{h}}\left(\int_{\mathfrak{o}_{1}}^{\mathfrak{o}_{2}}|\xi(q)|^{z} d q\right)^{\frac{1}{z}} .
\end{aligned}
$$

To show the sharpness and exactness of the constant $\left(|\xi(q)|^{z} d q\right)^{\frac{1}{z}}$, let us discover a function $\phi$ for which the inequality in (24) is gained. For $1<\mathfrak{h}<\infty$ take $\phi$ such that

$$
\phi^{n}(q)=\operatorname{sgn} \xi(q)|\xi(q)|^{\frac{1}{\mathfrak{h}-1}}
$$

For $\mathfrak{h}=\infty$ take $\phi^{n}(q)=\operatorname{sgn} \xi(q)$, for $\mathfrak{h}=1$ we prove that

$$
\left|\int_{\mathfrak{o}_{1}}^{\mathfrak{o}_{2}} \xi(q) \phi^{n}(q) d q\right| \leq \max _{q \in\left[\mathfrak{o}_{1}, \mathfrak{o}_{2}\right]}|\xi(q)|\left(\int_{\mathfrak{o}_{1}}^{\mathfrak{o}_{2}}\left|\phi^{n}(q)\right| d q\right)
$$


is the most suitable inequality. Assume that $|\xi(q)|$ acquires a maximum at $q_{o} \in\left[\mathfrak{o}_{1}, \mathfrak{o}_{2}\right]$. First, we suppose that $\xi\left(q_{o}\right) \geq 0$. For $\epsilon$ small enough, we determine $\phi_{\epsilon}(q)$ by

$$
\phi_{\varepsilon}(q):= \begin{cases}0, & \mathfrak{o}_{1} \leq q \leq q_{o}, \\ \frac{1}{\varepsilon n !}\left(q-q_{o}\right)^{n}, & q_{o} \leq q \leq q_{o}+\varepsilon \\ \frac{1}{n !}\left(q-q_{o}\right)^{n-1}, & q_{o}+\varepsilon \leq q \leq \mathfrak{o}_{2} .\end{cases}
$$

Then, for small enough $\epsilon$,

$$
\left|\int_{\mathfrak{o}_{1}}^{\mathfrak{o}_{2}} \xi(q) \phi^{n}(q) d q\right|=\left|\int_{q_{o}}^{q_{o}+\epsilon} \xi(q) d q\right|=\frac{1}{\epsilon} \int_{q_{o}}^{q_{o}+\epsilon} \xi(q) d q .
$$

Now, using inequality (25), we have,

$$
\frac{1}{\epsilon} \int_{q_{o}}^{q_{o}+\epsilon} \xi(q) d q \leq \xi\left(q_{o}\right) \int_{q_{o}}^{q_{o}+\epsilon} \frac{1}{\epsilon} d q=\xi\left(q_{0}\right)
$$

Since

$$
\lim _{\epsilon \rightarrow 0} \frac{1}{\epsilon} \int_{q_{o}}^{q_{o}+\epsilon} \xi(q) d q=\xi\left(q_{o}\right)
$$

this statement follows $\xi\left(q_{o}\right) \leq 0$

$$
\phi_{\varepsilon}(q):= \begin{cases}\frac{1}{n !}\left(q-q_{o}-\varepsilon\right)^{n-1}, & \mathfrak{o}_{1} \leq q \leq q_{o}, \\ -\frac{1}{\varepsilon n !}\left(q-q_{o}-\varepsilon\right)^{n}, & q_{o} \leq q \leq q_{o}+\varepsilon, \\ 0, & q_{o}+\varepsilon \leq q \leq \mathfrak{o}_{2} .\end{cases}
$$

and the rest of the proof also follows the same steps as above.

For further results we denote

$$
\zeta(q)=\int_{\mathfrak{o}_{1}}^{\mathfrak{o}_{2}} J(G(g(b), r)) G_{n-2}(r, q) d r \geq 0, \quad q \in\left[\mathfrak{o}_{1}, \mathfrak{o}_{2}\right]
$$

and

$$
\varphi(\zeta, \zeta)=\frac{1}{\mathfrak{o}_{2}-\mathfrak{o}_{1}} \int_{\mathfrak{o}_{1}}^{\mathfrak{o}_{2}} \zeta^{2}(q) d q-\left(\frac{1}{\mathfrak{o}_{2}-\mathfrak{o}_{1}} \int_{\mathfrak{o}_{1}}^{\mathfrak{o}_{2}} \zeta(q) d q\right)^{2}
$$

Theorem 3.6 If $\left(.-\mathfrak{o}_{1}\right)\left(\mathfrak{o}_{2}-.\right)\left[\phi^{n+1}\right]^{2} \in L\left[\mathfrak{o}_{1}, \mathfrak{o}_{2}\right]$ with the assumptions of Theorem 2.5 , then we have

$$
\begin{aligned}
J(\phi(g))= & \sum_{\mathfrak{z}=0}^{\mathfrak{u}} \frac{\phi^{(\mathfrak{z}+2)}\left(\mathfrak{o}_{1}\right)}{\mathfrak{z} !} \int_{\mathfrak{o}_{1}}^{\mathfrak{o}_{2}} J(G(g(b), r))\left(r-\mathfrak{o}_{1}\right)^{\mathfrak{z}} d r \\
& +\sum_{d=0}^{n-\mathfrak{u}-4}\left[\sum_{\mathfrak{z}=0}^{d} \frac{(-1)^{d-\mathfrak{z}}\left(\mathfrak{o}_{2}-\mathfrak{o}_{1}\right)^{d-\mathfrak{z}} \phi^{(\mathfrak{u}+3+d)}\left(\mathfrak{o}_{2}\right)}{(\mathfrak{u}+1+\mathfrak{z}) !(d-\mathfrak{z}) !}\right]
\end{aligned}
$$




$$
\begin{aligned}
& \times \int_{\mathfrak{o}_{1}}^{\mathfrak{o}_{2}} J(G(g(b), r))\left(r-\mathfrak{o}_{1}\right)^{(\mathfrak{u}+1+\mathfrak{z})} d r \\
& +\frac{\phi^{n-1}\left(\mathfrak{o}_{2}\right)-\phi^{n-1}\left(\mathfrak{o}_{1}\right)}{\mathfrak{o}_{2}-\mathfrak{o}_{1}} \int_{\mathfrak{o}_{1}}^{\mathfrak{o}_{2}} \zeta(q) d q+\kappa_{n}\left(\mathfrak{o}_{2}, \mathfrak{o}_{1} ; \phi\right) .
\end{aligned}
$$

While the remainder $\kappa_{n}\left(\mathfrak{o}_{2}, \mathfrak{o}_{1} ; \phi\right)$ satisfies the bound

$$
\left|\kappa_{n}\left(\mathfrak{o}_{2}, \mathfrak{o}_{1} ; \phi\right)\right| \leq \sqrt{\frac{\mathfrak{o}_{2}-\mathfrak{o}_{1}}{2}}[\varphi(\zeta, \zeta)]^{\frac{1}{2}}\left|\int_{\mathfrak{o}_{1}}^{\mathfrak{o}_{2}}\left(q-\mathfrak{o}_{1}\right)\left(\mathfrak{o}_{2}-q\right)\left[\phi^{(n+1)}(q)\right]^{2} d q\right|^{\frac{1}{2}}
$$

Proof The inequality (26) can be obtained in a similar way as the inequality (20).

Theorem 3.7 If $\phi^{n+1} \geq 0$ on $\left[\mathfrak{o}_{1}, \mathfrak{o}_{2}\right]$ with the assumptions of Theorem 2.5 , then equation (26) and the remainder $\kappa_{n}\left(\mathfrak{o}_{2}, \mathfrak{o}_{1} ; \phi\right)$ satisfies the condition

$$
\left|\kappa_{n}\left(\mathfrak{o}_{2}, \mathfrak{o}_{1} ; \phi\right)\right| \leq\left(\mathfrak{o}_{2}-\mathfrak{o}_{1}\right)\left\|\zeta^{\prime}\right\|_{\infty}\left\{\frac{\phi^{n-1}\left(\mathfrak{o}_{2}\right)+\phi^{n-1}\left(\mathfrak{o}_{1}\right)}{2}-\frac{\phi^{n-2}\left(\mathfrak{o}_{2}\right)-\phi^{n-2}\left(\mathfrak{o}_{1}\right)}{\mathfrak{o}_{2}-\mathfrak{o}_{1}}\right\}
$$

Proof The inequality (27) can be obtained in a similar way as the inequality (22).

Theorem 3.8 Let $\mathfrak{h}, z \in[1, \infty]$ such that $\frac{1}{\mathfrak{h}}+\frac{1}{z}=1$. If $\left|\phi^{n}\right|^{\mathfrak{h}}:\left[\mathfrak{o}_{1}, \mathfrak{o}_{2}\right] \rightarrow \mathbb{R}$ is integrable for some $n \in \mathbb{N}$ and $n \geq 4$ and the assumptions of Theorem 2.5 hold, then we obtain

$$
\begin{aligned}
J(\phi) & -\sum_{\mathfrak{z}=0}^{\mathfrak{u}} \frac{\phi^{(\mathfrak{z}+2)}\left(\mathfrak{o}_{1}\right)}{\mathfrak{z} !} \int_{\mathfrak{o}_{1}}^{\mathfrak{o}_{2}} J(G(g(b), r))\left(r-\mathfrak{o}_{1}\right)^{\mathfrak{z}} d r \\
& -\sum_{d=0}^{n-\mathfrak{u}-4}\left[\sum_{\mathfrak{z}=0}^{d} \frac{(-1)^{d-\mathfrak{z}}}{\left(\mathfrak{o}_{2}-\mathfrak{o}_{1}\right)^{d-\mathfrak{z}}} \phi^{(\mathfrak{u}+3+d)}\left(\mathfrak{o}_{2}\right)(\mathfrak{u}+1+\mathfrak{z}) !(d-\mathfrak{z}) !\right] \\
& \times \int_{\mathfrak{o}_{1}}^{\mathfrak{o}_{2}} J(G(g(b), r))\left(r-\mathfrak{o}_{1}\right)^{(\mathfrak{u}+1+\mathfrak{z})} d r \\
\leq & \left\|\phi^{(n)}\right\|_{\mathfrak{h}}\left(\int_{\mathfrak{o}_{1}}^{\mathfrak{o}_{2}}\left|\int_{\mathfrak{o}_{1}}^{\mathfrak{o}_{2}} J(G(g(b), r)) G_{n-2}(r, q) d r\right|^{z} d q\right)^{\frac{1}{z}} .
\end{aligned}
$$

The constant of (28) in the above equation on the right-hand side is sharp for $1 \leq \mathfrak{h} \leq \infty$ and the better estimate for $\mathfrak{h}=1$.

Proof The inequality (28) can be obtained in a similar way as the inequality (24).

\section{Conclusion}

The purpose of this paper is to obtain new refinements of Jensen's inequality and functionals on time scales. As Jensen's inequality is considered an important tool for producing classical inequalities, many classical inequalities can be improved by using the refined Jensen inequality. Also, new inequalities can be rewritten for several particular cases of time-scales integrals. Moreover, a similar method can be applied for the functionals obtained from the converse of Jensen's inequality and the Jensen-Mercer inequality. 


\section{Funding}

This research did not receive any specific grant from funding agencies in the public, commercial, or not-for-profit sectors.

\section{Availability of data and materials}

Data sharing is not applicable to this article as no datasets were generated or analyzed during the current study.

\section{Declarations}

\section{Competing interests}

The authors declare that they have no competing interests.

\section{Authors' contributions}

All authors jointly worked on the results and they read and approved the final manuscript.

\section{Author details}

${ }^{1}$ Department of Mathematics, Abbottabad University of Science and Technology, Havelian, Abbottabad, Pakistan.

${ }^{2}$ Department of Mathematics, University of Sargodha, Sargodha, Pakistan. ${ }^{3}$ Rudn University, 6 Miklukho-Maklay St, Moscow, 117198, Russia.

\section{Publisher's Note}

Springer Nature remains neutral with regard to jurisdictional claims in published maps and institutional affiliations.

Received: 24 May 2021 Accepted: 22 December 2021 Published online: 12 January 2022

\section{References}

1. Agarwal, R.P., Bohner, M., Peterson, A.: Inequalities on time scales: a survey. Math. Inequal. Appl. 4, 535-558 (2001)

2. Agarwal, R.P., Wong, P.J.Y.: Error Inequalities in Polynomial Interpolation and Their Applications. Kluwer Academic, Dordrecht (1993)

3. Alomari, M.: q-Benoulli inequality. Turk. J. Sci. 3(1), 32-39 (2018)

4. Bibi, R., Nosheen, A., Pecaric, J.: Generalization of Jensen-type linear functional on time scales via Lidstone polynomial. Cogent Math. Stat. 4(1), 1330670 (2017)

5. Bibi, R., Nosheen, A., Pecaric, J.: Extended Jensen's type inequalities for diamond integrals via Taylor's formula. Turk. J. Inequal. 3(1), 7-18 (2019)

6. Bohner, M., Peterson, A.: Dynamic Equations on Time Scales: An Introduction with Applications. Springer, Berlin (2001)

7. Bohner, M., Peterson, A.: Advances in Dynamic Equations on Time Scales. Springer, Berlin (2003)

8. Brito da Cruz, A.M.C., Martins, N., Torres, D.F.M.: The diamond integrals on time scales. Bull. Malays. Math. Sci. Soc. 38(4), 1453-1462 (2015)

9. Cerone, P., Dragomir, S.S.: Some new Ostrowski-type bounds for the Čebyšev functional and applications. J. Math. Inequal. 8, 159-170 (2014)

10. Davis, P.J.: Interpolation and Approximation. Courier Corporation, United States (1975)

11. Ekinci, A.: Inequalities for convex functions on time scales. TWMS J. Appl. Eng. Math. 9(1), 64-72 (2019)

12. Guessab, A.: Direct and converse results for generalized multivariate Jensen-type inequalities. J. Nonlinear Convex Anal. 13(4), 777-797 (2012)

13. Guessab, A., Schmeisser, G.: Sharp integral inequalities of the Hermite-Hadamard type. J. Approx. Theory 115(2), 260-288 (2002)

14. Guessab, A., Schmeisser, G.: Convexity results and sharp error estimates in approximate multivariate integration. Math. Compet. 73(247), 1365-1384 (2004)

15. Guessab, A., Schmeisser, G.: Sharp error estimates for interpolatory approximation on convex polytopes. SIAM J. Numer. Anal. 43(3), 909-923 (2005)

16. Özkan, U.M., Sarikaya, M.Z., Yildirim, H.: Extensions of certain integral inequalities on time scales. Appl. Math. Lett. 21(10), 993-1000 (2008)

17. Pečarić, J.E., Tong, Y.L.: Convex Functions, Partial Orderings, and Statistical Applications. Academic Press, San Diego (1992)

18. Sheng, Q., Fadag, M., Henderson, J., Davis, J.M.: An exploration of combined dynamic derivatives on time scales and their applications. Nonlinear Anal., Real World Appl. 7(3), 395-413 (2006)

19. Whittaker, J.: Interpolatory Function Theory. Cambridge Tracks in Math. (1935)

20. Wong, P.J.: Abel-Gontscharoff boundary value problems on measure chains. J. Comput. Appl. Math. 142(2), 331-355 (2002)

21. Wong, P.J.: Optimal Abel-Gontscharoff interpolation error bounds on measure chains. J. Comput. Appl. Math. 141(1-2), 267-282 (2002)

22. Wong, P.J.: Abel-Gontscharoff interpolation: continious, discrete and time scale. J. Comput. Appl. Math. 164, 763-782 (2004) 